\title{
Double Protection of the Higgs Potential in a Supersymmetric Little Higgs Model
}

\author{
Zurab Berezhiani, ${ }^{1}$ Piotr H. Chankowski, ${ }^{2}$ Adam Falkowski, ${ }^{2,3}$ and Stefan Pokorski ${ }^{2,4}$ \\ ${ }^{1}$ Dipartimento di Fisica, Università di L'Aquila, and Laboratori Nazionali del Gran Sasso, 67010 Assergi AQ, Italy \\ ${ }^{2}$ Institute of Theoretical Physics, Warsaw University, Hoża 69, 00-681 Warsaw, Poland \\ ${ }^{3}$ DESY, Notkestrasse 85, 22607 Hamburg, Germany \\ ${ }^{4}$ CERN Theory Division, CERN, Geneva 23, Switzerland \\ (Received 14 October 2005; published 25 January 2006)
}

\begin{abstract}
A mechanism of double protection of the Higgs potential, by supersymmetry and by a global symmetry, is investigated in a class of supersymmetric models with the $S U(3)_{C} \times S U(3)_{W} \times U(1)_{X}$ gauge symmetry. The electroweak symmetry can be then broken with no fine-tuning at all.
\end{abstract}

DOI: 10.1103/PhysRevLett.96.031801

PACS numbers: 12.60.Jv, 11.15.Ex

Stabilizing the Higgs potential is the central motivation for most extensions of the standard model (SM). In softly broken supersymmetry, the quadratic sensitivity of the Fermi scale to the ultraviolet (UV) cutoff is removed to all orders in perturbation theory. In generic supersymmetric models, the Higgs potential mass parameter depends quadratically on the soft supersymmetry breaking scale $M_{\text {soft }}$ and logarithmically on the cutoff $\Lambda_{\mathrm{UV}}$. For example, in the minimal supersymmetric standard model (MSSM), the one-loop corrections which lead to the electroweak symmetry breaking are dominated by the top sector contribution, and one approximately has

$$
m_{H}^{2} \approx m_{0}^{2}-\left(3 / 8 \pi^{2}\right) y_{t}^{2} M_{\mathrm{soft}}^{2} \ln \left(\Lambda_{\mathrm{UV}}^{2} / M_{\mathrm{soft}}^{2}\right),
$$

where $y_{t}$ is the top quark Yukawa coupling. This mechanism of radiative electroweak symmetry breaking strongly links the electroweak scale with $M_{\text {soft }}$. Indeed, the treelevel term $m_{0}^{2}$ contains a supersymmetry breaking contribution of order $M_{\text {soft }}^{2}$ (and the $\mu$-term contribution, which should be of the same order as $M_{\text {soft }}$ ), and, for $\Lambda_{\mathrm{UV}}$ close to the grand unification scale, the one-loop term is also of that order. However, in view of the existing experimental constraints, such a relation appears to be unsatisfactory. The lower limit on the Higgs boson mass and the precision electroweak data puts a lower bound on $M_{\text {soft }}$ of order of $1 \mathrm{TeV}$. In consequence, since $m_{H}^{2} \approx-M_{Z}^{2} / 2$, the cancellation between the tree-level and one-loop terms in the Higgs potential must be at least 1 part to 100 - the fact known as the "supersymmetric fine-tuning problem."

The following two features would improve this picture. The underlying physics should forbid the tree-level Higgs boson mass parameter. This would also have an advantage of avoiding the $\mu$ problem of the MSSM. Second, $\Lambda_{\mathrm{UV}}$ in (1) should be replaced by another scale of order $M_{\text {soft }}$. This would lead to $m_{H}^{2} \sim M_{\text {soft }}^{2} / 16 \pi^{2}$ and the correct value of $m_{H}^{2}$ for $M_{\text {soft }}$ of order $1 \mathrm{TeV}$.

The supersymmetric fine-tuning problem has stimulated several authors to look for alternatives to supersymmetry. The little Higgs models [1] revive the idea of the Higgs doublet being a pseudo Goldstone boson of some global symmetry spontaneously broken at a scale $\mathcal{O}(1 \mathrm{TeV})$. However, the scale of its breaking is usually linked to the mass scale of new gauge bosons, $W^{ \pm \prime}$ and/or a $Z^{\prime}$, and is constrained by precision electroweak data. In consequence, the fine-tuning in the Higgs potential is at least as large as in supersymmetric models [2,3].

In this Letter, we explore the idea of double protection of the Higgs potential. This mechanism operates in supersymmetric models in which the Higgs doublet is a pseudo Goldstone boson of an approximate global symmetry spontaneously broken at a scale $f \sim 1 \mathrm{TeV}$. The additional symmetry forbids the tree-level Higgs boson mass term and, together with supersymmetry, removes also the logarithmic dependence of $m_{H}^{2}$ on $\Lambda_{\mathrm{UV}}$ at one loop: $\Lambda_{\mathrm{UV}}$ gets replaced there with the mass scale of an additional heavy top quark which is of order $f$. As a result, the dominant one-loop contribution to $m_{H}^{2}$ is of the form

$$
\begin{aligned}
m_{H}^{2} \approx & -\left(3 / 8 \pi^{2}\right) y_{t}^{2}\left[\left(M_{\text {soft }}^{2}+f^{2}\right) \ln \left(M_{\text {soft }}^{2}+f^{2}\right)\right. \\
& \left.-M_{\text {soft }}^{2} \ln M_{\text {soft }}^{2}-f^{2} \ln f^{2}\right] .
\end{aligned}
$$

This is finite and vanishes in the limit of unbroken supersymmetry $M_{\text {soft }} \rightarrow 0$ as well as for unbroken global symmetry $f \rightarrow 0$.

The idea of double protection has been explored in Ref. [4] in a model proposed in Ref. [5]. However, in that model the scale of the $S U(3)$ symmetry breaking is also linked to the mass of the $Z^{\prime}$ boson. The allowed parameter space is then very limited, and the fine-tuning remains as large as in the MSSM. Here we discuss the mechanism of double protection in a class of models in which the global $S U(3)$ is a natural consequence of a $S U(3)$ gauge symmetry and the scales $F$ and $f$ of spontaneous gauge and global symmetry breaking, respectively, are unrelated. Therefore, experimental limits on the masses of new gauge bosons do not constrain $f$, and, for $f \leqslant$ $1 \mathrm{TeV} \ll F$, the electroweak symmetry can be broken with no fine-tuning at all.

We consider a class of supersymmetric models with $S U(3)_{C} \times S U(3)_{W} \times U(1)_{X}$ gauge symmetry. The electroweak $S U(2) \times U(1)_{Y}$ group is a subgroup of 
$S U(3)_{W} \times U(1)_{X}$, and the matter and Higgs fields are extended to $S U(3)_{W}$ multiplets. Several models of this kind exist in the literature [6,7], and others can be constructed. They differ in the assignment of particles to $S U(3)_{W} \times U(1)_{X}$ representations as well as in existence of additional exotic matter multiplets. The assignment can be such that all anomalies cancel $[6,7]$. Below, we concentrate only on the most universal features of such models.

We shall require that the Higgs sector has global symmetry $S U(3)_{1} \times S U(3)_{2}$ whose diagonal subgroup is the gauge $S U(3)_{W}$ group. To this end, we choose the Higgs sector consisting of two pairs of Higgs multiplets: $\Phi_{D}$, $\Phi_{U}$ - a triplet and an antitriplet of $S U(3)_{1}$-and $\mathcal{H}_{d}$, $\mathcal{H}_{u}$-a triplet and an antitriplet of $S U(3)_{2}$. The Higgs multiplets should acquire vacuum expectation values (VEVs) aligned in such a way that the $S U(2)$ gauge symmetry remains unbroken:

$$
\begin{aligned}
\left\langle\Phi_{D}\right\rangle^{T} & =\left(0,0, F_{D}\right), & \left\langle\mathcal{H}_{d}^{T}\right\rangle & =(0,0, f \cos \beta), \\
\left\langle\Phi_{U}\right\rangle & =\left(0,0, F_{U}\right), & \left\langle\mathcal{H}_{u}\right\rangle & =(0,0, f \sin \beta) .
\end{aligned}
$$

For $F_{U}, F_{D} \gg f$, we get the following picture. The $S U(3)_{W}$ gauge and the global $S U(3)_{1}$ symmetries are broken down to $S U(2)$ at the scale $F$, while the global $S U(3)_{2}$ survives and is spontaneously broken only at the scale $f$. This pattern leads to ten Goldstone bosons, five of which become longitudinal components of the massive gauge bosons corresponding to broken $S U(3)_{W}$ generators. The five physical Goldstone bosons are dominantly linear combinations of the components of $\mathcal{H}_{u}$ and $\mathcal{H}_{d}$. They can be conveniently parametrized as follows:

$$
\begin{aligned}
& \mathcal{H}_{u}=f \sin \beta\left(\frac{H^{\dagger}}{|H|} \sin (|H| / f), e^{(i \eta / f \sqrt{2})} \cos (|H| / f)\right), \\
& \mathcal{H}_{d}^{T}=f \cos \beta\left(\frac{H}{|H|} \sin (|H| / f), e^{(i \eta / f \sqrt{2})} \cos (|H| / f)\right) .
\end{aligned}
$$

Here $H$ is a weak $S U(2)$ doublet, which is identified with the SM Higgs doublet, and $|H|=\sqrt{H^{\dagger} H}$. The remaining Goldstone boson $\eta$ is a SM singlet. We will comment on its physical effects at the end of the Letter.

We are interested in a scenario in which the global symmetry breaking scale $f$ is $f \sim 1 \mathrm{TeV}$. Obviously, supersymmetry should not be broken spontaneously at the scale $F$. The required pattern can be obtained by choosing the following superpotential for the Higgs sector [8]:

$$
W=\lambda_{1} N_{1}\left(\Phi_{U} \Phi_{D}-\mu^{2}\right)+\lambda_{2} N_{2} \mathcal{H}_{u} \mathcal{H}_{d}+\kappa_{2} N_{2}^{3},
$$

where we introduced singlet superfields $N_{1,2}$ and $\mu$ is a mass parameter. Note that the terms $\Phi_{U} \mathcal{H}_{d}$ and/or $\mathcal{H}_{u} \Phi_{D}$ are not present as they would break the global $S U(3)_{1} \times S U(3)_{2}$ symmetry. In supersymmetric models, the form of the superpotential is radiatively stable due to the nonrenormalization theorem, even if it is not the most general one consistent with the gauge symmetry. Its constrained form could be a consequence of the local symmetry structure of the high energy completion or of some discrete symmetries, but in this Letter we do not construct any explicit models which would ensure this.

The superpotential (5) is a simple choice with the necessary qualitative features. In the limit of unbroken supersymmetry, the scalar potential resulting from (5) has the minimum for $F_{U}=F_{D}=\mu$ and vanishing other VEVs. The soft SUSY breaking terms shift $F_{U}$ and $F_{D}$ by terms of order $M_{\text {soft }}^{2} / F$, so that $F_{U}^{2}-F_{D}^{2} \sim M_{\text {soft }}^{2}$. To study the dynamics of light fields, one integrates the heavy components of $\Phi_{U}, \Phi_{D}$, and $N_{1}$. This yields the potential for $\mathcal{H}_{u}$, $\mathcal{H}_{d}$, and $N_{2}$ consisting (up to terms of order $1 / F^{2}$ ) of the $F$-term part following from (5), soft terms, quartic $D$ terms corresponding to the unbroken $S U(2) \times U(1)_{Y}$ symmetry generators [analogous terms corresponding to the broken generators of $S U(3)_{W} \times U(1)_{X}$ cancel out when the heavy fields are decoupled], and a term [9]

$$
\Delta M^{2}\left[c_{1}\left(H_{d}^{\dagger} H_{d}-H_{u}^{\dagger} H_{u}\right)+c_{2}\left(\left|S_{d}\right|^{2}-\left|S_{u}\right|^{2}\right)\right],
$$

where $\Delta M^{2}=M_{U}^{2}-M_{D}^{2}$ is the difference of soft masses of $\Phi_{U}\left(\Phi_{D}\right), H_{u, d}\left(S_{u, d}\right)$ are the doublet (singlet) components of $\mathcal{H}_{u, d}$, and $c_{1}, c_{2}$ are numerical factors. The term (6), which remains after the heavy fields are decoupled, would make the mass terms of $\mathcal{H}_{u}$ and $\mathcal{H}_{d}$ not $S U(3)_{2}$ symmetric and no light doublet would be present. To have it, we require $M_{D}^{2} \approx M_{U}^{2}$ at the $F$ scale. Below the scale $F$, the only explicit $S U(3)_{2}$ breaking terms arising from original $D$ terms are then the quartic $S U(2) \times U(1)_{Y} D$ terms.

Spontaneous breaking of the global $S U(3)_{2}$ symmetry can be induced by the soft masses $m_{u}, m_{d}$, and $m_{n}$ of $\mathcal{H}_{u}$, $\mathcal{H}_{d}$, and $N_{2}$, respectively. In particular, for very large $\tan \beta$ and neglecting trilinear soft terms, we find

$$
\begin{aligned}
& f^{2} \approx-m_{n}^{2} / \lambda_{2}^{2}, \quad\left\langle N_{2}\right\rangle^{2} \approx-m_{u}^{2} / \lambda_{2}^{2}, \\
& \tan \beta \approx-\left(\lambda_{2} / \kappa_{2}\right)\left(m_{n}^{2}+m_{u}^{2}-m_{d}^{2}\right) / m_{u}^{2} .
\end{aligned}
$$

For generic soft masses, $\tan \beta \gg 1$ is obtained for $\left|\lambda_{2}\right| \gg$ $\left|\kappa_{2}\right|$. The negative value of $m_{u}^{2}$ may result, as in the MSSM, from the renormalization group running. $m_{n}^{2}$ becomes negative also due to the Yukawa interaction if $\left|\lambda_{2}\right| \sim 1$. For $m_{u}^{2}<0$, the minimum (7) is only a local one [for $\left\langle N_{2}\right\rangle=\left\langle S_{d}\right\rangle=0,\left\langle S_{u}\right\rangle \rightarrow \infty$, the potential of the low energy theory is unbounded from below and is stabilized only by the $D$ terms of the original $S U(3)_{W}$ gauge group]. One has to assume that the Universe is in the metastable minimum (7). Since the tunneling to the true minimum usually takes longer than the Universe's lifetime, this is a viable assumption. For moderate values of $\tan \beta \geqslant 5$, complete formulas analogous to (7) show that there are solutions with $m_{u}^{2}>0$ and $m_{d}^{2} \gtrsim m_{u}^{2} \tan ^{2} \beta$, and the minimum is then the global one.

Since the global $S U(3)_{2}$ symmetry is broken by the $S U(2) \times U(1)_{Y}$ quartic $D$ terms, $H$ is a pseudo Goldstone boson. We require that another source of the explicit breaking comes from the supersymmetric interactions in the top sector. The Higgs doublet can acquire then a negative mass parameter at one-loop level. However, due to an interplay 
between the approximate global symmetry and supersymmetry, these radiative corrections are finite at one loop [4]. Logarithmic divergences are cut off by the contribution of the additional top quarks, whose presence is required by the approximate $S U(3)$. This double protection mechanism alleviates the supersymmetric fine-tuning problem, as we demonstrate in the following.

We shall illustrate our point in a specific model, which is a supersymmetrization of "the simplest little Higgs" model of Ref. [6] and comment on the generality of our results later. The relevant chiral fermion superfields are the $S U(3)_{W}$ triplet $\Psi_{Q}=(Q, T)^{T}$ and the $S U(3)_{W}$ quark singlets $t^{c}$ and $T^{c}$. The superpotential is given by

$$
W=y_{1} \Phi_{U} \Psi_{Q} T^{c}+y_{2} \mathcal{H}_{u} \Psi_{Q} t^{c} .
$$

As for the Higgs fields, this is not the most general choice consistent with the gauge symmetry. Once the $S U(3)_{W}$ gauge symmetry is broken at the scale $F$, the second term in Eq. (8) preserves the global $S U(3)_{2}$ symmetry, while the first term breaks it explicitly.

Inserting the parametrization (4) of $\mathcal{H}_{u, d}$, we find the top matrix $\mathcal{L}=-(t, T) \mathcal{M}_{\text {top }}\left(t^{c}, T^{c}\right)$ as a function of the VEV of $H$. For $|H| \ll f$, it has two eigenvalues corresponding to the top quark and its heavy $S U(3)_{W}$ partner:

$$
\begin{gathered}
m_{t} \approx y_{t}|H|, \quad y_{t}=\frac{y_{1} y_{2} F}{m_{T}} \sin \beta, \\
m_{T} \approx \sqrt{y_{1}^{2} F^{2}+y_{2}^{2} f^{2} \sin ^{2} \beta} .
\end{gathered}
$$

Let us summarize the orders of magnitudes of the model parameters needed for a coherent picture. The scale $F$ cannot be too high, because soft terms must be approximately $S U(3)$ symmetric at the $\mathrm{TeV}$ scale. For definiteness, we will assume $F \sim 10 \mathrm{TeV}$. On the other hand, to minimize the fine-tuning, we will need $m_{T} \sim 1 \mathrm{TeV}$, i.e., $y_{1} F \sim y_{2} f \lesssim 1 \mathrm{TeV}$. Note that small $y_{1}$ is consistent with our requirement that renormalization group effects do not significantly split $M_{U}^{2}$ and $M_{D}^{2}$. For given values of $F$ and $f$, the Yukawa couplings $y_{1}$ and $y_{2}$ can be chosen such that the standard model top Yukawa coupling $y_{t}$ has the correct value and $m_{T}$ is in the desired range. Relation (9) implies then a lower limit $m_{T}>2 y_{t} f$.

We move to the determination of the Higgs potential

$$
V=\delta m_{H}^{2}|H|^{2}+\left(\lambda_{0}+\delta \lambda\right)|H|^{4}+\cdots .
$$

The tree-level quartic term $\lambda_{0}|H|^{4}$ comes from $S U(2) \times$ $U(1)_{Y} D$ terms. For $\tan \beta \gg 1, \lambda_{0}=\left(g^{2}+g_{y}^{2}\right) / 8$. The one-loop corrections $\delta m_{H}^{2}$ and $\delta \lambda$ can be computed from the one-loop effective potential:

$$
\Delta V_{\text {one-loop }}=\frac{1}{64 \pi^{2}} \mathrm{~S} \operatorname{Tr}\left\{\mathcal{M}^{4}\left(\ln \frac{\mathcal{M}^{2}}{\Lambda_{\mathrm{UV}}^{2}}-\frac{3}{2}\right)\right\} .
$$

By computing $\mathrm{S} \operatorname{Tr} \mathcal{M}^{4}$, it can be seen that there is no logarithmically divergent contribution from the top and stop sector to the Higgs potential mass parameter $\delta m_{H}^{2}$. This can be understood by a simple dimensional analysis.
The coefficient of a logarithmically divergent term would have to break both supersymmetry and the approximate global symmetry, but in the top-stop sector there is no such dimensionful parameter. The conclusion holds for any stop soft masses and trilinear terms, as long as their tree-level values respect the $S U(3)_{2}$ global symmetry. Furthermore, in a simplified situation when all stops have approximately the same soft mass squared $m_{Q}$ and the mixing between left- and right-chiral stops is negligible, we obtain a simple formula for $\delta m_{H}^{2}$ :

$$
\begin{aligned}
\delta m_{H}^{2} \approx & -\frac{3}{8 \pi^{2}} y_{t}^{2}\left[\left(m_{Q}^{2}+m_{T}^{2}\right) \ln \left(m_{Q}^{2}+m_{T}^{2}\right)-m_{Q}^{2} \ln m_{Q}^{2}\right. \\
& \left.-m_{T}^{2} \ln m_{T}^{2}\right]+\Delta .
\end{aligned}
$$

Here $\Delta$ stands for contributions from other sectors of the theory. For example, gauginos contribute

$$
\Delta \supset \frac{1}{8 \pi^{2}}\left(3 g_{2}^{2} M_{2}^{2}+g_{y}^{2} M_{y}^{2}\right) \ln \left(F / M_{\text {soft }}\right),
$$

where $M_{2, y}$ are gaugino masses. The cutoff is set by the scale at which the $S U(3)_{W}$ gauge symmetry is restored.

The top contribution in (12) has a remarkable property that it vanishes for both $m_{Q} \rightarrow 0$ and $m_{T} \rightarrow 0$. As advertised, the double protection mechanism leads to the softening of the UV sensitivity of the Higgs potential. For a given $m_{T}$, the top contribution is minimized for $m_{Q}=m_{T}$, while for $m_{Q}>m_{T}$ it increases only as $\ln \left(m_{Q} / m_{T}\right)$.

The dominant contribution to $\delta \lambda$ is given by

$$
\begin{aligned}
\delta \lambda \approx & \frac{3}{16 \pi^{2}} y_{t}^{4}\left\{\ln \left(m_{T}^{2} m_{Q}^{2}\right)-\ln \left(m_{Q}^{2} m_{t}^{2}+m_{T}^{2} m_{t}^{2}\right)+\frac{3}{2}\right. \\
& \left.-2\left(m_{Q}^{2} / m_{T}^{2}\right)\left[\ln \left(m_{Q}^{2}+m_{T}^{2}\right)-\ln \left(m_{Q}^{2}\right)\right]\right\} .
\end{aligned}
$$

For $m_{Q} \sim m_{T}$, it behaves as $\left(3 / 8 \pi^{2}\right) y_{t}^{4} \ln \left(m_{Q} / m_{t}\right)$. Therefore, the physical Higgs boson mass given by

$$
M_{h}^{2}=2\left(\lambda_{0}+\delta \lambda\right) v^{2}=M_{Z}^{2}+2 \delta \lambda v^{2}
$$

takes values similar as in the MSSM.

We now estimate the level of fine-tuning in the electroweak breaking. The value of $\delta m_{H}^{2}$ is determined by the relation $v^{2}=-\delta m_{H}^{2} /\left(\lambda_{0}+\delta \lambda\right)$, where $v=246 \mathrm{GeV}$. The correct $v$ can always be obtained by arranging for appropriate $\Delta$ (e.g., by tuning the gaugino masses), but large cancellations between the top contribution and $\Delta$ are unnatural. We quantify the fine-tuning (FT) as follows:

$$
\mathrm{FT}=\left|\frac{\Delta-\left|\delta m_{H}^{2}\right|}{\left|\delta m_{H}^{2}\right|}\right|=\left|\frac{\Delta-\frac{1}{2} M_{h}^{2}}{\frac{1}{2} M_{h}^{2}}\right| .
$$

In Fig. 1, we show the dependence of the Higgs boson mass $M_{h}$ and the fine-tuning on the input parameters of our model. $M_{h}$ is plotted as a function of $m_{T}$ for $m_{Q}=m_{T}$ (the dependence on the $m_{Q} / m_{T}$ ratio is very weak in this case). The experimental limit $M_{h}>114.4 \mathrm{GeV}$ is also shown. For $m_{t}$, we have used the $\overline{\mathrm{MS}}$ value $164 \pm 3 \mathrm{GeV}$ corresponding to physical $m_{t}=172.7 \pm 2.9 \mathrm{GeV}$ [10]. The 

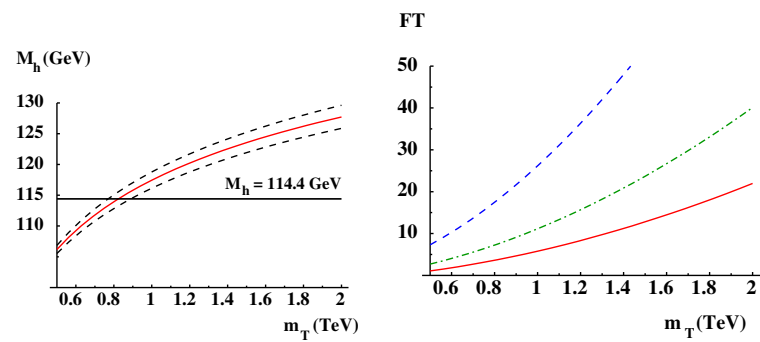

FIG. 1 (color online). Left panel: The Higgs boson mass as a function of $m_{T}$ for $m_{Q} / m_{T}=1$. The dashed lines indicate the effect of the $2 \sigma$ uncertainty of the top mass. Right panel: The fine-tuning as a function of $m_{T}$ for $m_{Q} / m_{T}=1$ (solid curve), $m_{Q} / m_{T}=2$ (dotted-dashed curve), and $m_{Q} / m_{T}=10$ (dashed curve).

effect of varying $m_{t}$ within the $2 \sigma$ limit is also displayed. The fine-tuning as a function of $m_{T}$ is plotted for several values of the ratio $m_{Q} / m_{T} \geq 1$ (the formulas are symmetric under the interchange of $m_{Q}$ and $m_{T}$ ). We see that, for $0.8 \mathrm{TeV}<m_{T}<1 \mathrm{TeV}$ and $m_{Q} \sim m_{T}$, the electroweak symmetry can be broken with no fine-tuning at all, while the experimental Higgs boson mass bound can be respected. For $m_{T}>1 \mathrm{TeV}$, the fine-tuning is of order $10 \%$. Note that, even when either $m_{Q}$ or $m_{T}$ are of order $10 \mathrm{TeV}$, fine-tuning is not worse than in the MSSM with $\mathrm{TeV}$ scale stop masses.

Finally, we point out the main experimental signatures of the double protection mechanism. One is the existence of a heavy top quark with mass $\sim 1 \mathrm{TeV}$. New gauge bosons are heavier, not within the reach of the LHC. The gauginos should weigh $\sim 1 \mathrm{TeV}$ at most. Furthermore, if the scale $f$ is linked to the soft supersymmetry breaking parameters, as in the mechanism discussed in this Letter, squark masses are around $1 \mathrm{TeV}$, too. However, one can perhaps invent other mechanisms of generating the scale $f$. It is worth noting that, merely from the point of view of the electroweak symmetry breaking with little fine-tuning, the squark masses are very weakly constrained.

The singlet $\eta$ in the parametrization (4) is a true massless Goldstone boson of the broken Peccei-Quinn $U(1)$ symmetry acting on the $S U(3)$ partners of the SM weak doublets. As such, it is subject to experimental, cosmological, and astrophysical constraints on light pseudoscalars. However, $\eta$ couples to the ordinary matter only via mixing of the latter with their $S U(3)$ partners. For the first two generations, such mixing is very small, as long as the corresponding $S U(3)$ partners have masses of order $f$. Therefore, processes such as energy loss in stars put on $f$ only a weak lower bound of order $100 \mathrm{GeV}$.

The model studied above can be extended to accommodate all three generations of quark and leptons and their masses [6]. Anomaly cancellation implies then that their assignment to $S U(3)_{W} \times U(1)_{X}$ representations cannot be generation universal. Furthermore, it is not possible to embed this spectrum in a simple unified group. However, one can consider models with a different spectrum which ensure the double protection mechanism for the Higgs potential. For example, with the $S U(3)_{C} \times S U(3)_{W} \times$ $U(1)_{X}$ gauge symmetry, the following matter content can be chosen (the first two generations can be introduced analogously): $\Phi_{U}, \mathcal{H}_{u}:(1,3)_{1 / 3} ; \Phi_{D}, \mathcal{H}_{d}:(1, \overline{3})_{-1 / 3} ;$ $\psi_{Q}^{T}=\left(Q_{3}, D\right):(3,3)_{0} ; T^{c}:(\overline{3}, 3)_{-1 / 3} ; T:(3, \overline{3})_{1 / 3} ; t^{c}:$ $(\overline{3}, 1)_{-2 / 3} ; \quad b_{1}^{c}, b_{2}^{c}: \quad(\overline{3}, 1)_{1 / 3} ; \quad \psi_{L}^{T}=\left(L_{3}, E\right): \quad(1,3)_{-2 / 3} ;$ $\tau_{1}^{c}, \tau_{2}^{c}:(1,1)_{1}$ with the superpotential

$$
\begin{aligned}
W= & y_{1} \Phi_{U} \Psi_{Q} T^{c}+y_{2} \mathcal{H}_{u} T t^{c}+\mu_{T} T T^{c}+y_{b 1} \psi_{Q} \mathcal{H}_{d} b_{1}^{c} \\
& +y_{b 2} \psi_{Q} \Phi_{D} b_{2}^{c}+y_{\tau 1} \psi_{L} \mathcal{H}_{d} \tau_{1}^{c}+y_{\tau 2} \psi_{L} \Phi_{D} \tau_{2}^{c} .
\end{aligned}
$$

The top sector here is slightly more complicated. It contains a vectorlike triplet pair $T$ and $T^{c}$ with a supersymmetric mass term $\mu_{T}$. Still, for $F \gg f$, the picture is qualitatively and quantitatively the same as in the supersymmetric version of the model of Ref. [6] discussed in this Letter. In particular, the formulas (12) and (14) for $\delta m_{H}^{2}$ and $\delta \lambda$ still hold, with $y_{1} F$ replaced by $\mu_{T}$ in Eq. (9). This indicates that the structure of the effective Higgs potential at one loop is a general feature of models in which the double protection mechanism is realized.

In conclusion, the double protection of the Higgs potential by supersymmetry softly broken at the $\mathrm{TeV}$ scale and by a global symmetry which is spontaneously broken at the scale $\lesssim 1 \mathrm{TeV}$ may be a mechanism allowing one to understand the origin and the stability of the Fermi scale.

P. H. Ch., A. F., and S. P. were partially supported by the European Community Contract No. MRTN-CT-2004503369 for years 2004-2008 and by the Polish KBN Grant No. 1 P03B 09929 for years 2005-2007.

[1] N. Arkani-Hamed, A. G. Cohen, and H. Georgi, Phys. Lett. B 513, 232 (2001); N. Arkani-Hamed, A. G. Cohen, E. Katz, A.E. Nelson, T. Gregoire, and J. G. Wacker, J. High Energy Phys. 08 (2002) 021; N. Arkani-Hamed, A. G. Cohen, E. Katz, and A.E. Nelson, J. High Energy Phys. 07 (2002) 034.

[2] J. A. Casas, J. R. Espinosa, and I. Hidalgo, J. High Energy Phys. 03 (2005) 038.

[3] G. Marandella, C. Schappacher, and A. Strumia, Phys. Rev. D 72, 035014 (2005).

[4] P.H. Chankowski, A. Falkowski, S. Pokorski, and J. Wagner, Phys. Lett. B 598, 252 (2004).

[5] A. Birkedal, Z. Chacko, and M. K. Gaillard, J. High Energy Phys. 10 (2004) 036.

[6] M. Schmaltz, J. High Energy Phys. 08 (2004) 056.

[7] P. Frampton, Phys. Rev. Lett. 69, 2889 (1992); F. Pisano and V. Pleitez, Phys. Rev. D 46, 410 (1992); D. E. Kaplan and M. Schmaltz, J. High Energy Phys. 10 (2003) 039; W. Skiba and J. Terning, Phys. Rev. D 68, 075001 (2003).

[8] P. Fayet, Nucl. Phys. B90, 104 (1975).

[9] A. Pomarol and S. Dimopoulos, Nucl. Phys. B453, 83 (1995); R. Rattazzi, Phys. Lett. B 375, 181 (1996).

[10] The CDF and D0 Collaborations and the Tevatron Electroweak Working Group, hep-ex/0507091. 